albumin decreases the respiratory rate and added palmitate returns it to higher levels.

In new-born and adult rats neither albumin nor palmitate has any effect (Table).

The age difference cannot be explained by a different fatty acid content because the amount of free fatty acids/mg of mitochondrial protein in new-born animals is about $60 \%$ higher than in 6 - to 30 -day-old rats $(120$ to $140 \mathrm{nmmole} / \mathrm{mg}$ protein) and adult animals have approximately the same amount of fatty acids bound to the mitochondria as 6- to 30 -day-old rats.

Our results show that for maximum activation of the respiratory rate in uncoupled brown-fat mitochondria both ATP and carnitin are necessary. The inhibitory effect of serum albumin indicates that endogenous fatty acids are oxidized. Evidently the endogenous fatty acids are bound to the external mitochondrial membrane, where they are activated ${ }^{9}$ and transported as carnitine esters into the mitochondria. Apparently, in our experiments, mitochondria contain such a large amount of endogenous fatty acids that addition of palmitate has no further activating effect on mitochondrial respiration.
Zusammenfassung. Nachweis der endogenen Atmungsaktivierung von Mitochondrien im braunen Fettgewebe durch ATP und Karnitin. Eine maximale Aktivierung wird bei $6-30$ Tage alten Ratten gefunden, während sie in neugeborenen und erwachsenen Tieren kleiner ist. Nur bei den 6-30 Tage alten Tieren hemmt Albumin die durch ATP und Karnitin erhöhte Atmung.

\section{Z. Drahota, EVA Honoví and P. HAHN}

Institute of Physiology, Caechoslovak Academy of

Sciences, Praha (Czechoslovakia),

21 September 1967.

9 K. R. Norum, M. Farstad and J. Bremer, Biochem. Biophys. Res. Comm. 24, 797 (1966).

\title{
Uber die Hydrierung im Ringsystem ungesättigter Steroide mit Mycobacterium phlei
}

In unseren früheren Arbeiten 1,2 wurden zahlreiche Oxydationsprozesse beschrieben, die mit Hilfe eines Mycobakteriumstammes durchgeführt werden können. Durch $\mathrm{Zu}-$ gabe von 8-Hydroxychinolin kann der Abbau des Ringsystems verhindert werden; dadurch häufen sich $\Delta 1,4-3-$ Keto-Verbindungen mit Androstangerüst an.

In der vorliegenden Arbeit berichten wir über einige Beobachtungen der enzymatischen Hydrierung des Ringsystems ungesättigter Steroide. In der Literatur fanden wir Angaben über die mikrobielle Sättigung der Doppelbindungen $\Delta 1^{3}, \Delta 4^{4,6}, \Delta 6^{6}, \Delta 7^{1,7}$, über die Umwandlung von $\Delta 16$-20-Ketosteroiden in 17-iso-Steroide ${ }^{8}$ und über den Seitenkettenabbau der letztgenannten Verbindungen ${ }^{9,10}$.

Wir untersuchten den Metabolismus einiger im Ring (B) ungesättigter, bzw. $\Delta 16-20$-Ketosteroide unter aeroben Bedingungen. Die Umsetzungen wurden mit einer in wässriger 0,4prozentiger Natriumchlorid-Lösung suspendierter Züchtung von Mycobacterium phlei bei $37^{\circ} \mathrm{C}$ durchgeführt, welche vorher in Maisquellwasser-GlycerinMedium anwuchs und anschliessend vom Medium getrennt wurde. Die Substrat- und 8-Hydroxychinolin-Konzentrationen betrugen $400 \mu \mathrm{g} / \mathrm{ml}$, bzw. $100 \mu \mathrm{g} / \mathrm{ml}$. Nach $48 \mathrm{~h} \mathrm{In-}$ kubation wurden die Kulturfiltrate der einzelnen Ausgangsmaterialien mit Chloroform extrahiert und das 8Hydroxychinolin mit verdünnter, wässriger Oxalsäure entfernt. Schliesslich wurden die Extrakte im Vakuum eingedampft. Im Rückstand konnten wir Androsta-1,4dien-3,17-dion und geringe Mengen von Androst-4-en3,17-dion als Umsetzungsprodukte nachweisen. Durch Kristallisation bzw. präparative Dünnschichtchromatographie wurde aus den folgenden Substraten Androsta-1,4dien-3,17-dion erhalten. Ausbeuten (in \% der Theorie): Androsta-4, 6-dien-3, 17-dion (60\%), Pregna-4, 6-dien3, 20-dion (46\%), Cholesta-4,6-dien-3-on (32\%), Ergosta$4,6,22$-trien-3-on $(31 \%), 5 \alpha$-Cholest-7-en-3 $\beta$-ol $(25 \%)$, Cholesta-5, 7 -dien-3 $\beta$-ol (26\%), $5 \alpha$-Ergosta-7, 22-dien-3 $\beta$ ol $(29 \%)$, Ergosta-5, 7, 22-trien-3 $\beta$-ol $(26 \%)$, Pregna-5, 16dien-3 $\beta$-ol-20-on (75\%). Auch die Ester (Acetate, Ben- zoate) der letztgenannten 5 Verbindungen wurden in Androsta-1,4-dien-3,17-dion übergeführt.

Wie man auf Grund der Versuche feststellen kann, ist es möglich, die Doppelbindungen $\Delta 6$ und $\Delta 7$ unter aeroben Umständen mit Mycobacterium phlei zu sättigen, sowie 116-20-Keto-Steroide in 17-Ketosteroide $z \mathfrak{u}$ uberführen. Das aus den verschiedenen Ausgangsmaterialien isolierte Androsta-1,4-dien-3,17-dion ist ein wertvoller Ausgangsstoff für die Östron- und Norsteroid-Synthesen.

Summary. Androsta-1, 4-diene-3, 17-dione was obtained from $\Delta 6-, \Delta 7$ - and $\Delta-16$-steroids by Mycobacterium phlei. The breakdown of the steroid nucleus was inhibited by 8 hydroxyquinoline added to the culture medium.

\section{G. Ambrus, E.TömörkÉNY und K.G. BükI}

Institut für Arzneimittelforschung,

Budapest 4 (Ungarn), 13. November 1967.

${ }^{1}$ G. WIX, K.G.Bükx, E.Tömörkeny und G.Amrrus, Ungarisches Patent No. 153831 (1965).

2 G.Wix, K.G.Bukr, E.Tömörkêny und G.Ambrus, Steroids, im Druck.

3 A.Butenandi, H.Dannenberg und L.A.Suranyi, Chem. Ber. $73,818(1940)$

${ }^{4}$ L. Mamolt, R. Koch und H.Teschen, Z. physiol. Chem. 261, 287 (1939).

5 L.Mamoli und G.Schramm, Chem. Ber. 71, 2698 (1938).

- Y.Y.Tsong, K.C. Wang und CH. J. Sim, Biochim. biophys. Acta 93, 398 (1964).

7 R.Deghenghi, S. Rakhit, K.Singh, C.Vezina und Ch.J.Sih, Steroids 10, 313 (1967).

P.D.Meister, D.H.Peterson, H.C.Murray, S.H.Eppstein, L.M. Reine Ke, A. Weintraue und H. M. Leigh, J. Am. chem. Soc. 75,55 (1953).

${ }^{9}$ G. Wtx und M. Rados, Schweizer Patent, No. 376499 (1959).

10 O. EL-TAYEB, S.G. KNIGHT und CH. J.Sin, Biochim. biophys. Acta 93,411 (1964). 